\title{
Patient attitudes to type specific serological tests in the diagnosis of genital herpes
}

\author{
I Fairley, E F Monteiro
}

Objective: To assess patient attitudes to HSV-2 serotesting and the effect of providing detailed information regarding genital herpes, the blood test, and its implications.

Methods: Consecutive genitourinary medicine clinic attenders were asked to complete an anonymous self administered questionnaire. Half received minimal additional information while the other half received a detailed information sheet.

Results: Overall, 200 clinic attenders with a median age of 27 years (range 15-57) completed the questionnaire, $92.4 \%$ wanted to know if they, and $90.8 \%$ if their partners, had been infected with genital herpes; $65 \%$ expected testing as part of screening without further discussion. Overall, on a scale of $1-10,2 \%$ scored 1 (equivalent to definitely not wanting a test), while $45.5 \%$ scored 10 (equivalent to definitely wanting a test). The overall median score was 9 (95\% confidence interval 8-10) suggesting a strong opinion in favour of testing. The desire to test in each of five described hypothetical situations increased significantly $(p<0.001)$ when compared with the general desire.

Conclusions: Clinic attenders expressed a strong preference to be serotested for HSV-2 which was unaltered by the provision of information highlighting implications, although influenced significantly by the context in which they were asked. Should reliable tests become available the level of demand could have important implications on laboratory and counselling resources.

(Genitourin Med 1997;73:259-262)

Keywords: genital herpes; serology; patient attitudes

\section{Introduction}

Two serotypes of herpes simplex virus (HSV) are recognised; both can cause cutaneous and mucocutaneous lesions, with the proportion attributable to HSV types 1 and 2 varying geographically. HSV-1 is common in the general population and often acquired non-sexually in childhood. HSV-2 is rarely a cause of oral lesions and serological evidence of HSV-2 is a good marker of genitally acquired infection. ${ }^{1}$ Currently, diagnosis relies on viral culture from clinically visible lesions. Commercially available serological tests cannot distinguish between past infection with HSV-1 and infection with HSV-2. Reliable type specific assays are available in only a few centres on a research basis.
Table 1 Patient characteristics

\begin{tabular}{lll}
\hline & No information & Information \\
\hline Age, median (range) & 27 (16-57) & 26 (15-51) \\
Number of sexual partners & 6 & 4 \\
1 & 4 & 10 \\
2 & 28 & 23 \\
$3-5$ & 19 & 24 \\
$6-10$ & 42 & 38 \\
$>10$ & & \\
Partner status & 38 & 35 \\
Regular partner: & 18 & 9 \\
$\quad$ Living separately & 22 & 34 \\
$\quad$ Married & 22 & 21 \\
$\quad$ Co-habiting & $30 / 61$ & $42 / 53$ \\
No regular partner & $8 / 92$ & $16 / 84$ \\
Sense of risk of having acquired & & \\
Prenital herpes (yes/no) & & \\
by a doctor (yes/no) & & \\
\hline
\end{tabular}

Type specific assays may soon be marketed and will be useful on a population level to monitor changes in sexual behaviour but their role in clinical practice remains much debated. Other tests which have become available in recent years which have serious implications for the individual, such as HIV antibody testing and tests for genetic defects, have demonstrated that many factors impinge on the decision whether to be tested or not. This has led to the principle of informed consent for tests which have medical, psychological, or social implications. It is recognised that symptomatic genital herpes has significant psychosexual morbidity. ${ }^{2}$ If testing was widely performed, a large group of people will be subjected to the knowledge of a potentially lifelong, albeit in most cases, trivial but incurable infection that they can transmit to sexual partners. Opportunities for intervention include alterations in sexual lifestyle and consistent use of condoms during penetrative sexual contact. Long term suppressive antiviral treatment may also prove to be of some benefit. ${ }^{3}$ While doctors continue to argue the usefulness of these assays, no information regarding patient attitudes to HSV antibody testing is available.

In order to obtain informed opinion, detailed information regarding genital herpes, the blood test, and its implications would need to be given. This study assessed patient opinion by a structured self administered questionnaire. Two groups were identified. One group received the minimal additional information 


\begin{tabular}{|c|c|c|c|c|}
\hline & & $\begin{array}{l}\text { No information } \\
(n=100)\end{array}$ & $\begin{array}{l}\text { Information } \\
(n=100)\end{array}$ & p Value $(95 \%$ CI) \\
\hline \multirow{2}{*}{\multicolumn{2}{|c|}{$\begin{array}{l}\text { (1) If you had been infected with genital herpes } \\
\text { would you want to know (yes/no) } \\
\text { (2) If your partner had been infected with genital herpes } \\
\text { would you want to know (yes/no) }\end{array}$}} & $88 / 10$ & $94 / 5$ & $0.19(-0.13 \text { to } 0.02)^{\star}$ \\
\hline & & $85 / 13$ & $95 / 5$ & $0.05(-0.16$ to -0.003$)$ \\
\hline (3) & $\begin{array}{l}\text { If the blood test for genital herpes was available now, } \\
\text { would you }\end{array}$ & & & \\
\hline & Expect testing as part of a full infection screen & 64 & 64 & \\
\hline & Not want to be tested. & 2 & 2 & \\
\hline & Have a test only if advised by a doctor & $\begin{array}{l}9 \\
5\end{array}$ & $\begin{array}{l}8 \\
6\end{array}$ & $0.987 t$ \\
\hline & Tested only at your or your own doctor's request & 17 & 20 & \\
\hline
\end{tabular}

${ }^{\star} \chi^{2}$.

needed to provide consent for the study, while the other group was given a detailed information sheet before completing the questionnaire in order to establish whether baseline attitudes were altered by the provision of detailed information.

\section{Methods}

Consecutive clinic attenders were asked to complete a questionnaire. Once verbal consent was obtained two groups were identified. Those with an even clinic number were given no additional information while those with an odd number were given a detailed three page information sheet to read before completing the questionnaire. The information sheet provided information relating to aspects of herpes virology, recognition of disease, modes of transmission, prevention, and treatment. The aim was to explain in language which patients could understand that herpes virus could establish an incurable infection which may lead to apparent or unapparent recurrent episodes which would render the person intermittently and unpredictably infectious to sexual partners or (in the case of women) neonates. Treatment options ranging from no treatment to continuous suppressive therapy were explained. Emphasis was placed on the widespread and covert nature of HSV in the general population. That two types of HSV exist and the differences between them were explained. Herpes antibody testing and details regarding the known facts and uncertainties about the implications of testing positive for either HSV-1 or HSV-2 (particularly with

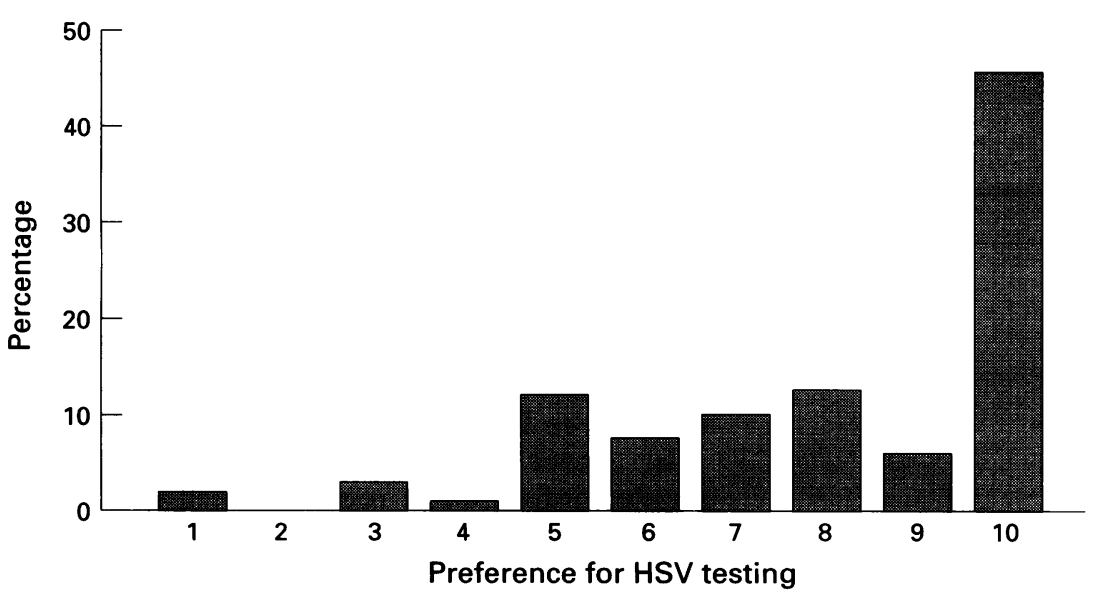

Preference for HSV antibody testing on a scale of 1-10 ( $n=200) .(1=$ definitely do not want a test; 10 = definitely do want a test.) regard to sexual transmission and pregnancy), or testing negative for both were explained. In particular, patients were informed that the presence of HSV-2 antibody essentially con- $\stackrel{\mathbb{2}}{\circ}$ firms genital herpes while its absence does not के exclude genital infection with HSV-1, $\overrightarrow{0}$ although the majority with antibodies to the latter would have perioral infection.

The questionnaire initially asked for details regarding age, sex, number of lifetime sexual partners and current partner status, previous is diagnosis of genital herpes, and whether any of previous partner had documented infection. Patients were then asked whether they would want to know if either their partner or they $\vec{D}$ themselves had genital herpes. Respondents' overall desire to test generally and in specific $\stackrel{c}{\stackrel{c}{+}}$ situations (for example, partner known to have genital herpes, symptoms suggestive of genital herpes, if a current partner had developed a : first episode of genital herpes, or if pregnant) was assessed using a scale of $1-10$ (1 = definitely not wanting a test, $10=$ definitely wanting a test). Assessment was also made of $\frac{\otimes}{\Phi}$ whether patients expected testing as part of a $\stackrel{\vec{Z}}{\vec{Z}}$ routine screen, at their own request, or on the recommendation of a doctor and how much prior discussion was felt necessary before testing.

Ethics committee approval was obtained for the study. Results were analysed using STATA statistical software. Categorical variables were $\delta$ compared using Fisher's exact test, $\chi^{2}$, MannWhitney $\mathrm{U}$, or Wilcoxon matched pairs sign $\frac{\mathrm{O}}{3}$ rank test as appropriate, using $5 \%(\mathrm{p}<0.05)$ as the significance level.

\section{Results}

One hundred consecutive male and 100 consecutive female clinic attenders consenting to participate completed the questionnaire. Patient characteristics, separated into those $\stackrel{\mathscr{Q}}{\rightarrow}$ who received the detailed information and $T$ those who did not, are shown in table 1 . Age, $\stackrel{\circ}{\mathbb{D}}$ lifetime numbers of sexual partners, current partner status, and perception of risk of having acquired genital herpes for each group was equivalent.

Table 2 shows the proportion of patients wanting to know if they or their partners had been infected with genital herpes separated according to whether or not they received the additional information sheet. Table 2 also shows the proportions in these groups expecting testing to be initiated routinely; at their 
Table 3 Preferences for HSV serotest in men and women in specific situations

\begin{tabular}{|c|c|c|c|c|}
\hline Situation & $\begin{array}{l}\text { All patients } \\
\text { Median } \\
(95 \% \text { CI })\end{array}$ & $\begin{array}{l}\text { No information } \\
\text { Median } \\
\text { (10-90th centile) }\end{array}$ & $\begin{array}{l}\text { Information } \\
\text { Median } \\
\text { (10-90th centile) }\end{array}$ & p Value \\
\hline (1) Generally & $9(8-10)$ & $10(5-10)$ & $8(5-10)$ & $0 \cdot 44$ \\
\hline $\begin{array}{l}\text { (2) If previous sex with a partner } \\
\text { known to have genital herpes }\end{array}$ & $10(10-10)$ & $10(8-10)$ & $10(8-10)$ & 0.89 \\
\hline (3) If symptoms suggestive of genital herpes & $10(10-10)$ & $10(9-10)$ & $10(9-10)$ & 0.63 \\
\hline genital herpes & $10(10-10)$ & $10(10-10)$ & $10(8-10)$ & 0.58 \\
\hline (5) Desire to test female partner if pregnant & $10(10-10)$ & $10(5-10)$ & $10(8-10)$ & 0.42 \\
\hline partner pregnant & $10(10-10)$ & $10(5-10)$ & $10(7-10)$ & 0.45 \\
\hline
\end{tabular}

p Values calculated using Mann-Whitney $U$ test.

Scale $1-10(1=$ definitely do not want a test, $10=$ definitely want a test $)$.

own request; at their doctors' request; at their own or their doctors' request; or not wanting to be tested at all. Borderline significance was reached only in the proportion wanting to know if their partner had been infected, with more of those receiving the additional information wanting to know.

Subjects were asked to rate the desirability of testing on a scale of 1-10 (1 = definitely not wanting a test and $10=$ definitely wanting a test) generally and in specific described hypothetical situations. The figure illustrates graphically the overall desire to be HSV-2 serotested with table 3 showing the median score for each of the described situations. For clinic attenders given additional information and no additional information, median scores with a range (10th-90th centiles) are given. For all patients together the median and $95 \%$ confidence interval are quoted. Comparison of these two groups using Mann-Whitney $U$ as a non-parametric test of equivalence showed no significant differences. Comparing each specific situation against the general desire to test by Wilcoxon matched pairs sign rank test, showed a highly significant increase in desire to test $(p<0.001)$ for each specified situation.

\section{Discussion}

The results of this study clearly demonstrate that patients attending the genitourinary medicine clinic in Leeds express a strong preference to be serotested for HSV-2 antibody, with only a $2 \%$ refusal rate for testing. The majority of patients $(65 \%)$ expected testing as part of a full infection screen without the need for further information. A recent audit looked at the uptake of available serological tests in 100 new patients attending our department. This showed that $28 \%$ had an HIV test, of which 18 had attended specifically for this reason so that $12 \%$ had an HIV test when not specifically attending for this reason. Of those attending and agreeing to full screening (with or without an $\mathrm{HIV}$ test), there was an $88 \%$ uptake for a serological test for syphilis which is offered routinely as part of our screening. Low et al studied the potential need for HIV counselling in the Leeds genitourinary medicine (GUM) and antenatal clinic populations. ${ }^{4}$ They found $11 \%$ of GUM attenders had been HIV tested, whereas $57 \%$ would have accepted the offer of a test, giving a $68 \%$ requirement (met and unmet demand) for HIV testing. Two thirds of offers of testing were accepted for "peace of mind". This all points to a high proportion of patients wanting serological tests if available and a high uptake if offered routinely.

A small number of clinic attenders declined to answer the questionnaire. We have no data on the opinions of this group but we feel that it is unlikely to have significantly affected the validity of the results. Provision of a detailed information sheet did not significantly affect opinion, although borderline significance was reached with an increased number of participants receiving the information sheet wanting to know if their partner had been infected. This may suggest that much of the response is intuitive rather than reasoned judgment, although it would be important to know how much patients understood the complexity of the information provided. When piloting the information sheet with patients it was generally well received and understood by patients, although this was not formally tested. The questionnaire also asked if the information sheet answered all their questions; $85 \%$ said yes with few making any comments in the space provided. It would also be important to know whether detailed counselling or a "cooling off" period would have influenced decisions. Studies assessing screening for the cystic fibrosis gene for example, show that $70-95 \%$ accept offers of immediate testing whereas only $25 \%$ will make a return visit for such a test. ${ }^{5}$ No patient in our study requested further information and very few gave uncertain responses. Furthermore, opinion did not differ in the 24 patients previously diagnosed as having genital herpes (data not shown). This group having lived with all the implications of the diagnosis remain very much in favour of testing. The decision to be tested was significantly altered by the context in which individuals are asked to make that decision. If a previous partner was known to have herpes or was experiencing a first episode, if the individual had symptoms suggestive of herpes, or if one of the couple was pregnant, then the desire to test was even stronger.

Clinical and non-serological methods are poorly specific and insensitive determinants of past infection with HSV-2 when compared with type specific serological techniques. Asymptomatic infection is common and studies demonstrate a substantial burden of HSV-2 in the general population. ${ }^{6}$ Testing the individual would allow recognition of infection and the need to prevent transmission. Testing partners of those who have genital herpes to assess their 
risk of acquisition may help some and be a source of conflict to others. Pregnant women are an important group as they can transmit infection to the neonate with a potential for significant morbidity and mortality. For those already infected a positive test may lead to anxiety with minimal additional benefit in terms of intervention. However, primary genital herpes, especially in third trimester, can have important consequences. ${ }^{7}$ The identification of HSV negative women with serodiscordant partners would appear to be one of the most compelling medical reasons for testing, although cost effectiveness at the population level remains uncertain. ${ }^{8}$

No type specific assay has so far been successfully adapted for widespread commercial application but this will no doubt occur in the near future. As type specific tests become available the debate regarding their use will intensify. The ability to detect HSV-1 antibody provides little useful information as most will be non-sexually acquired perioral infections with only a proportion representing genital HSV and no easy way of distinguishing the two. What to tell the patient with HSV-1 antibody is, therefore, less clear. The presence of HSV-2 on the other hand strongly suggests genital infection and as most patients with HSV-2 antibody shed virus intermittently the implications are more clear. While HSV-2 essentially confirms genital herpes it does not exclude it and patients cannot be reassured that they do not have genital herpes. Physicians will need to carefully consider the management and therapeutic implications and potential pitfalls of testing. They must be clear what the test tells them and what they would advise their patients when the result is available. Patient opinion does need to be considered and we have shown a very strong patient opinion in favour of testing. This study did not assess patients' understanding of these complicated issues, the impact of counselling, or the time required to provide a level of understanding needed for informed consent. These remain areas of ongoing study. We also need to know whether such opinions exist at other geographical locations and clinical settings.
The usefulness of HSV-2 testing in any given clinical situation could be debated. However, if the limitations are kept in mind, there may be situations in which testing could prove useful-for example, undiagnosed recurrent genital ulcer disease. We believe it is premature to make HSV type specific antibody tests widely available when costs may be high and benefit to patients uncertain. If it became established that widespread testing is clinically useful a secondary consideration would be the resource implications. In view of the complexity of interpreting results and the possible post test psychological and psychosexual morbidity, extra facilities for counselling would be necessary. We have demonstrated a large potential demand highlighting the importance of assessing the laboratory and counselling resources needed to introduce routine type specific HSV serological testing to genitourinary medicine clinics. The patient will ultimately make the decision whether or not to have a test, if it is made available, but its introduction should be delayed. Until such information is available, we would only consider the introduction of type specific HSV-2 antibody testing in specific situations.

The authors would like to thank Susan Bogle for her statistica advice.

1 Corey $\mathrm{L}$. The current trend in genital herpes. Progress in prevention. Sex Transm Dis 1994;21(Suppl):S38-44.

2 Mindel A. Long-term clinical and psychological management of genital herpes. $f$ Med Virol 1993;1(Suppl): 39-44.

3 Wald A, Zeh J, Barnum G, Davis LG, Corey L. Suppression of subclinical shedding of herpes simplex virus type 2 with acyclovir. Ann Intern Med 1996;124:8-15.

4 Low ML, Lacey CJN, Buchan PC, Franks AJ, Waugh MA The potential requirement for HIV counselling and voluntary testing in women of childbearing age. Int $\mathcal{F} S T D$ AIDS 1993;4:155-8.

5 Harris H, Scotcher D, Hartley N, Wallace A, Craufurd D, Harris R. Cystic fibrosis carrier testing in early pregnancy by general practitioners. $B M F^{1993 ; 306: 1580-3}$

6 Cowan FM, Johnson AM, Ashley R, Corey L, Mindel A. Antibody to herpes simplex virus type 2 as serological Antibody to herpes simplex virus type 2 as serological marker of

7 Brown ZA, Vontver LA, Benedetti J, Critchlow CW, Sells CJ, Berry S, et al. Effects on infants of a first episode of genital herpes during pregnancy. $N$ Engl $f \mathrm{Med}$ 1987;317:1246-51

8 Kulhanjian JA, Soroush V, Au DS, Bronzan RN, Yasukawa LL, Weylman LE, et al. Identification of women a unsuspected risk of primary infection with herpes simplex virus type 2 during pregnancy. $N$ Engl f Med 1992; 326:916-20. 